\title{
Capturing and concentrating adenovirus using magnetic anionic nanobeads
}

\author{
This article was published in the following Dove Press journal: \\ International Journal of Nanomedicine \\ 9 May 2016 \\ Number of times this article has been viewed
}

\author{
Akikazu Sakudo' \\ Koichi Baba ${ }^{2}$ \\ Kazuyoshi Ikuta ${ }^{1,3}$ \\ 'Department of Virology, Research \\ Institute for Microbial Diseases, \\ Osaka University, Yamadaoka, Suita, \\ Osaka, Japan; ${ }^{2}$ Baba Pediatric Clinic, \\ Kadoma, Osaka, Japan; ${ }^{3}$ Kanonji \\ Institute, The Research Foundation \\ for Microbial Diseases of Osaka \\ University, Kanonji, Kagawa, Japan
}

Correspondence: Akikazu Sakudo Laboratory of Biometabolic Chemistry, School of Health Sciences, Faculty of Medicine, University of the Ryukyus, 207 Uehara, Nishihara,

Okinawa 903-0215, Japan

$\mathrm{Tel}+8|98895| 252$

$\mathrm{Fax}+8|98895| 252$

Email sakudo@med.u-ryukyu.ac.jp

\begin{abstract}
We recently demonstrated how various enveloped viruses can be efficiently concentrated using magnetic beads coated with an anionic polymer, poly(methyl vinyl ethermaleic anhydrate). However, the exact mechanism of interaction between the virus particles and anionic beads remains unclear. To further investigate whether these magnetic anionic beads specifically bind to the viral envelope, we examined their potential interaction with a nonenveloped virus (adenovirus). The beads were incubated with either adenovirus-infected cell culture medium or nasal aspirates from adenovirus-infected individuals and then separated from the supernatant by applying a magnetic field. After thoroughly washing the beads, adsorption of adenovirus was confirmed by a variety of techniques, including immunochromatography, polymerase chain reaction, Western blotting, and cell culture infection assays. These detection methods positively identified the hexon and penton capsid proteins of adenovirus along with the viral genome on the magnetic beads. Furthermore, various types of adenovirus including Types 5, 6, 11, 19, and 41 were captured using the magnetic bead procedure. Our bead capture method was also found to increase the sensitivity of viral detection. Adenovirus below the detectable limit for immunochromatography was efficiently concentrated using the magnetic bead procedure, allowing the virus to be successfully detected using this methodology. Moreover, these findings clearly demonstrate that a viral envelope is not required for binding to the anionic magnetic beads. Taken together, our results show that this capture procedure increases the sensitivity of detection of adenovirus and would, therefore, be a valuable tool for analyzing both clinical and experimental samples.
\end{abstract}

Keywords: anionic polymer, virus concentration, adenovirus, capture, magnetic beads, poly(methyl vinyl ether-maleic anhydrate)

\section{Introduction}

Adenoviruses, which belong to the family Adenoviridae, are nonenveloped viruses with an icosahedral nucleocapsid and double-stranded DNA genome. The Mastadenovirus genus infects mammals and includes human, simian, equine, bovine, porcine, ovine, canine, and opossum viruses. In humans, 51 distinct adenoviral serotypes have been identified that can cause a wide range of illnesses including respiratory infections, gastroenteritis, acute febrile pharyngitis, pharyngoconjunctival fever, and epidemic keratoconjunctivitis. ${ }^{1,2}$

Currently, the 51 serotypes of human adenovirus are classified into subgroups A-F, ${ }^{3}$ although recent studies have reported an additional new serotype. ${ }^{4}$ Each type of adenovirus infects via a different route ${ }^{5,6}$ as follows: respiratory tract infection is caused by Types 1-7 (subgroups B, C, and E); ocular infection is caused by Types 8, 19, and 37 (subgroup D); and urinary tract infection is caused by Types 11 and 21 (subgroup B). 
A common causative agent of infantile diarrhea is Types 40 and 41 adenovirus (subgroup $\mathrm{F}$ ).

Recent developments in immunochromatography have facilitated the convenient detection of adenovirus in biological samples, which has considerably improved clinical diagnosis..$^{7-9}$ Nonetheless, immunochromatography is sometimes insufficiently sensitive to detect low levels of adenovirus in clinical samples. ${ }^{9}$ Although the polymerase chain reaction (PCR) is a highly sensitive means of detecting adenovirus, the method is time consuming and is generally performed in the laboratory rather than the clinic. Thus, novel methods are needed for the early and sensitive detection of adenovirus in clinical samples.

Rapid and sensitive detection of adenoviruses is crucially important for both restricting the spread of disease and improving therapeutic outcome. Pretreatment of clinical samples to concentrate adenovirus considerably increases the sensitivity of viral detection. There are two major considerations when developing a novel technique to concentrate adenovirus; first, the method should be compatible with current detection procedures, and second, it should be simple and straightforward to perform. Several approaches have been used to increase the concentration of viruses from clinical samples to enhance the sensitivity of detection. ${ }^{10-12}$ For example, ultracentrifugation and polyethylene glycol (PEG)-mediated precipitation have been used to concentrate a number of different viruses, including adenovirus. Although ultracentrifugation is a well-established procedure for concentrating viruses, it is time consuming and can be incompatible with PCR (ie, increases the number of false-positives).$^{13,14}$ Moreover, while PEG precipitation of virus particles is simple and easy to perform, the PEG can sometimes interfere with the subsequent PCR amplification step. ${ }^{15}$ In addition, both ultracentrifugation and PCR reduce infectivity of viruses. An alternative approach for concentrating viruses in clinical samples is to use magnetic beads coated with molecules that efficiently bind viral particles such as organic chemicals and biomolecules. Indeed, we and other groups have reported that magnetic beads coated with an anionic polymer, poly(methyl vinyl ether-maleic anhydrate) (poly(MVE-MA)), can be used to efficiently capture different viral types. ${ }^{16,17}$ These include human immunodeficiency virus Type 1 (HIV-1), ${ }^{18}$ respiratory syncytial virus (RSV), ${ }^{19}$ Borna disease virus, ${ }^{20}$ influenza virus, ${ }^{21}$ and dengue virus, ${ }^{22}$ which are all enveloped viruses. In addition, antibodies targeting the viral envelope proteins have been shown to interfere with the binding of virus particles to the anionic magnetic beads. ${ }^{18,19}$ The results of this study imply that the anionic

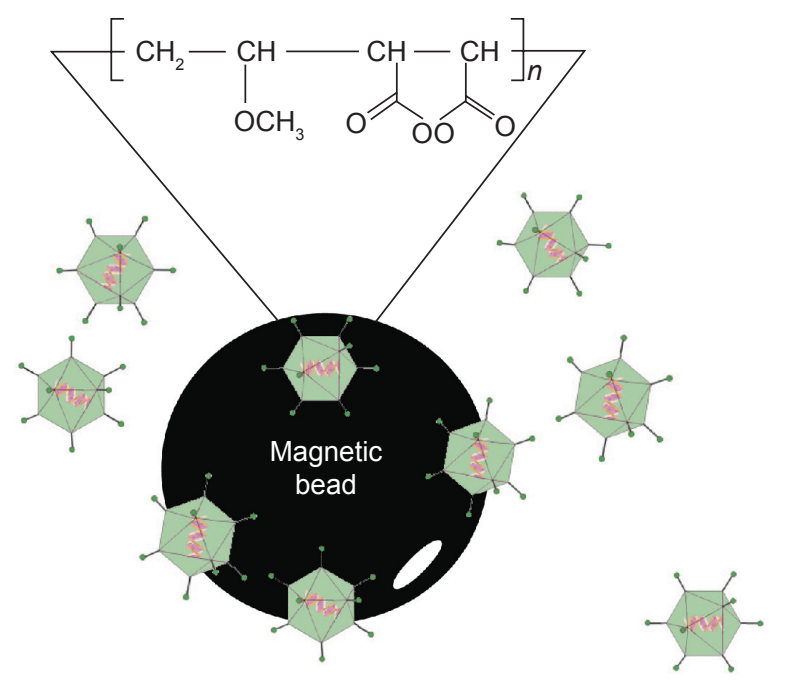

Figure I Schematic presentation of capturing adenovirus using magnetic anionic nanobead.

magnetic beads might bind viruses via the viral envelope, but it remains unclear whether the envelope is essential for the observed interaction. These considerations prompted us to further investigate whether the anionic magnetic beads are able to bind a nonenveloped virus.

Here, we determined whether anionic magnetic beads coated with poly(MVE-MA) are able to efficiently capture adenovirus, which is a representative nonenveloped virus (Figure 1). Furthermore, the applicability of this beadconcentrating method was examined using various types of adenovirus.

\section{Materials and methods Reagents}

Unless otherwise specified, chemical reagents were obtained from Sigma-Aldrich (St Louis, MO, USA) or Wako Pure Chemical Industries (Osaka, Japan). Magnetic particles (300 $\mathrm{nm}$ in diameter) with a high ferrite content were used in this study. The poly(MVE-MA) coating was grafted onto the magnetic particles in 5\% dimethyl sulfoxide and phosphate buffer for 3 hours at $37^{\circ} \mathrm{C}$ as described previously. ${ }^{16}$

\section{Adenovirus}

Purified recombinant adenovirus Type 6 (strain Tonsil99) was purchased from Hytest, Ltd. (Turku, Finland). Genetic recombinants of adenovirus Type 5, lacking the ability to grow, were purchased from Takara Bio Inc. (Otsu, Japan). Axcw2 is adenovirus Type 5 alone, while AxCAwt2 contains adenovirus Type 5, CAG promoter, cytomegalovirus enhancer, chicken $\beta$-actin promoter, and rabbit $\beta$-globin polyA site. AxCAiLacZ contains adenovirus Type 5, CAG 
promoter, cytomegalovirus enhancer, chicken $\beta$-actin promoter, rabbit $\beta$-globin polyA site, and Escherichia coli $\beta$-Gal gene (Takara Bio Inc.). Adenoviruses such as Adenoid75 (adenovirus Type 5, ATCC VR-5), Tonsil99 (adenovirus Type 6, ATCC VR-6), Slobitski (adenovirus Type 11, ATCC VR12), AV-587[3911] (adenovirus Type 19, ATCC VR-254), and Tak (73-3544) (adenovirus Type 41, ATCC VR930) were purchased from American Type Culture Collection (Manassas, VA, USA).

\section{Collection of nasal aspirates}

Clinical nasal aspirates were collected from pediatric patients at the Baba Pediatric Clinic (Osaka, Japan). The method used to collect the samples was described previously. ${ }^{17,23}$ Briefly, saline was introduced into the nasal cavity, and fluid was collected using a nasal aspirator, Belvital (Melisana, Nogent-sur-Marne, France). The nasal fluid was then filtered through a stainless steel mesh (200 grids per inch [25.4 mm]) to remove cell debris. The nasal aspirates were subsequently subjected to immunochromatography for adenovirus as described in the "Immunochromatography" section. As a negative control, nasal aspirates were also collected from healthy donors using the same procedure. The research project was approved by the Ethics Committee of the Research Institute for Microbial Diseases at Osaka University. Written informed consent was obtained from all the individuals who participated in this study.

\section{Capture of adenovirus}

Viral capture was performed as described previously. ${ }^{17}$ Briefly, the magnetic beads (volume of $50 \mu \mathrm{L}$ ) were washed twice with binding buffer and then twice more with phosphate-buffered saline (PBS). A $20 \mu \mathrm{L}$ aliquot of nasal aspirate or cell culture medium infected with adenovirus was diluted with $500 \mu \mathrm{L}$ of PBS and incubated with the washed magnetic beads for 20 minutes at room temperature. After applying a magnetic field, the supernatant was discarded. The beads were then washed three more times with PBS. Finally, the washed beads were resuspended in PBS and analyzed by Western blotting, immunochromatography, viral DNA extraction, or viral titration assay.

\section{Immunochromatography}

A rapid test for the detection of adenovirus antigens was performed using Quick Navi-Adeno (Denka Seiken Co., Ltd., Tokyo, Japan), which recognizes the adenovirus hexon protein. ${ }^{24}$ The experimental procedure was conducted according to the manufacturer's instructions.

\section{Enzyme-linked immunosorbent assay}

An adenovirus- $\mathrm{X}^{\mathrm{TM}}$ rapid titer kit (Clontech Laboratories Inc., Mountain View, CA, USA) was used to assay for adenovirus hexon protein. Briefly, samples were incubated with HEK293 cells for 48 hours, and the cells were then fixed with ice-cold 100\% methanol and stained with mouse anti-adenovirus hexon antibody. The signal was detected with a rat anti-mouse antibody conjugated to horseradish peroxidase and developed with metal-enhanced 3,3'diaminobenzidine tetrahydrochloride. Brown/black staining, indicating infection with adenovirus, was visualized by optical microscopy.

\section{Viral titration assay}

The number of plaque forming units (PFUs) per $\mathrm{mL}$ was determined by performing twofold serial dilutions of samples in 96-well plates containing HEK293 cells (ATCC CRL1573 $\left.{ }^{\mathrm{TM}}\right)$ as described in the manufacturer's instructions (Adeno- $\mathrm{X}^{\mathrm{TM}}$ Expression System User Manual, Clontech Laboratories Inc.). Prior to infection, cells were washed with PBS and then the infected cells were incubated at $37^{\circ} \mathrm{C}$ in an atmosphere of $5 \% \mathrm{CO}_{2}$ for 7 days. Virus titer was determined by counting the number of plaques. To minimize error, only plates containing between 10 and 100 plaques were counted (ie, depending on the size of the cell culture plate). According to statistical analysis, when 100 plaques are counted, the sample titer will vary by $\pm 10 \%$.

\section{Western blotting}

Each fraction was solubilized in an equal volume of $2 \times$ sodium dodecyl sulfate (SDS) gel-loading buffer $(90 \mathrm{mM}$ Tris- $\mathrm{HCl}$ [pH 6.8], 10\% mercaptoethanol, 2\% SDS, 0.02\% bromophenol blue, and 20\% glycerol), boiled for 5 minutes, and then resolved by SDS-polyacrylamide gel electrophoresis (PAGE) using an 8\% gel. The bands were then electroblotted onto a polyvinylidene difluoride (PVDF) membrane (Hybond-P; Amersham-Pharmacia Biotech, Piscataway, NJ, USA) for 60 minutes at $15 \mathrm{~V}$. The PVDF membrane was blocked with 5\% skimmed milk for 1 hour at room temperature and then incubated with goat anti-adenovirus polyclonal antibody (AP00664PU-N, Acris) in PBS containing $0.1 \%$ Tween 20 (PBS-T) and $0.5 \%$ skimmed milk for 1 hour at room temperature. After three washes with PBS-T, the membrane was incubated in PBS-T and $0.5 \%$ skimmed milk supplemented with anti-goat IgG conjugated to horseradish peroxidase (Jackson ImmunoResearch Laboratories, Inc., West Grove, PA, USA) for 1 hour at room temperature with gentle shaking. The membrane was 
then washed three times with PBS-T, and protein bands that reacted with the antibodies were visualized using an enhanced chemiluminescence detection kit (AmershamPharmacia Biotech). Chemiluminescence was detected using EzCaptureMG (ATTO Corp., Tokyo, Japan). Band intensity was analyzed by densitometric analysis with ImageJ software (National Institutes of Health).

\section{X-gal (5-bromo-3-indoyl- $\beta$-D- galactopyranoside) staining}

For X-gal staining, X-Gal Staining ${ }^{\mathrm{TM}}$ solution (Genlantis and Gene Therapy Systems, Inc, San Diego, CA, USA) was used. Briefly, cells were fixed with formaldehyde-glutaraldehyde buffer and washed with PBS. Samples were then incubated in X-gal staining solution for 18 hours at $37^{\circ} \mathrm{C}$ in a $\mathrm{CO}_{2}$ incubator. Blue cells, indicating infection with adenovirus, were visualized by optical microscopy.

\section{Viral DNA extraction and PCR amplification}

Adenovirus DNA was extracted using a QIAamp Viral RNA mini kit (Qiagen, Hilden, Germany). Viral nucleic acid derived from either the beads or an aliquot of each sample was extracted according to the manufacturer's instructions. DNA was extracted from the magnetic beads by adding lysis buffer prior to removing the beads. The DNA was then eluted into $60 \mu \mathrm{L}$ of nuclease-free water and amplified in a reaction mixture containing primers, Ex Taq (Takara bio Inc.) and $1 \times$ Ex Taq buffer. Amplification comprised 30 cycles of $94^{\circ} \mathrm{C}$ for 30 seconds, $50^{\circ} \mathrm{C}$ for 30 seconds, and $72^{\circ} \mathrm{C}$ for 2 minutes. PCR was carried out using the following primers with specificity for the adenovirus hexon gene:

Hexon-F: 5'-TGGGTGATAACCGTGTGCTA-3',

Hexon-R: 5'-TTAATGCTAGCCCCGTCAAC-3'.

The amplified DNA products were analyzed by agarose gel electrophoresis using a $1.2 \%$ gel.

\section{Real-time PCR}

Extracted viral DNA was also analyzed by real-time PCR using SYBR Premix Ex TaqII (Tli RNase H plus; Takara Bio Inc.) according to the manufacturer's instructions (Stratagene, La Jolla, CA, USA). Briefly, the real-time PCR components included SYBR Premix Ex Taq II and the forward and reverse target gene primers: realAdenoHexon-F, 5'-GACATGACTTTCGAGGTCGATCCCATGGA-3'; realAdenoHexon-R, 5'-CCGGCTGAGAAGGGTGTGCGC AGGTA-3'. Real-time PCR was performed using a Thermal Cycler Dice Real Time System (Takara Bio Inc.). The cycling program included initial denaturation at $95^{\circ} \mathrm{C}$ for 30 seconds, followed by 40 cycles of $95^{\circ} \mathrm{C}$ for 30 seconds and $60^{\circ} \mathrm{C}$ for 30 seconds. Each reaction was carried out in quadruplicate, and the results were analyzed using Thermal Cycler Dice Real Time System Single software (Takara Bio Inc.). The relative expression ratio of each sample was calculated using a mathematical model based on the amplification efficiency. PCR specificity was verified by dissociation curve analysis of the amplified DNA fragments of step $1\left(95^{\circ} \mathrm{C} / 15\right.$ seconds), step $2\left(60^{\circ} \mathrm{C} / 30\right.$ seconds), and step $3\left(95^{\circ} \mathrm{C} / 15\right.$ seconds $)$.

\section{DNA sequencing}

The products of conventional PCR and real-time PCR were purified and cloned into pT7Blue T-vector (Novagen, Madison, WI, USA). The identity of the PCR products was then verified by DNA sequencing on an ABI PRISM3100 Genetic Analyzer (Applied Biosystems, Foster City, CA, USA) using R-20mer primer and U-19mer primer (Novagen).

\section{Results}

Initially, we performed immunochromatography to verify whether the poly(MVE-MA)-coated anionic magnetic beads could efficiently capture adenovirus. For these experiments, recombinant adenovirus Type 6 (Tonsil99) was used as sample solution. Immunochromatography, employing an antibody against adenovirus hexon protein, was used to detect adenovirus in the bead fraction (BD; Figure 2). Adenovirus was also detected in samples containing the same quantity of adenovirus as in the BD (total fraction, TL). However, the corresponding band was absent in the supernatant after separation from the beads (SP; Figure 2). Compared with

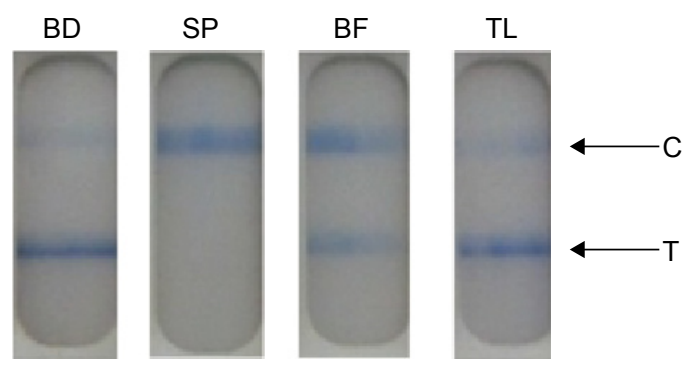

Figure 2 Detection of hexon protein of adenovirus adsorbed onto the anionic polymer-coated magnetic beads from diluted recombinant adenovirus Type 6. Notes: Recombinant adenovirus Type 6 (Tonsil99; $20 \mu \mathrm{L}, 0.05 \mathrm{mg} / \mathrm{mL}$ ) was diluted with PBS $(500 \mu \mathrm{L})$ and then incubated with anionic magnetic beads coated with anionic polymer, poly(MVE-MA). Quick Navi-Adeno was used for the detection of adenovirus capsid protein, hexon by immunochromatography. The presence of adenovirus hexon protein was interpreted on the basis of the presence or absence of a test line (T). A positive control was also included (C). Samples were divided into the following: BD, sample before incubation with the beads (BF), supernatant after incubation with the beads (SP), and samples containing the same quantity of adenovirus as in the BD (total fraction, $\mathrm{TL}$ ). In each case, samples were solubilized with lysis buffer and subjected to immunochromatography for detection.

Abbreviations: BD, bead fraction; PBS, phosphate-buffered solution; poly(MVE-MA), poly(methyl vinyl ether-maleic anhydrate). 


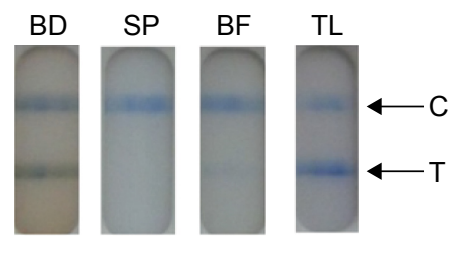

Adenoid75
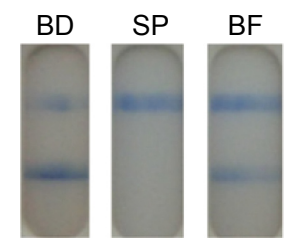

Tonsil99
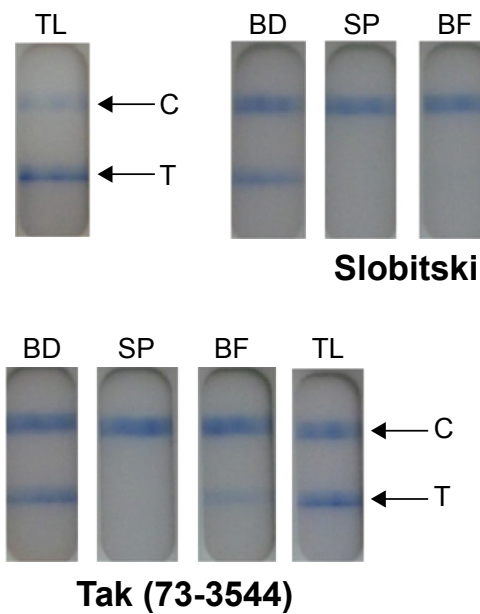

Figure 3 Adsorption of various types of adenovirus onto anionic polymer-coated magnetic beads.

Notes: Various strains of adenovirus including Adenoid75 (adenovirus Type 5), Tonsil99 (adenovirus Type 6), Slobitski (adenovirus Type II), AV-587[39II] (adenovirus Type 19), and Tak(73-3544) (adenovirus Type 4I) were diluted with PBS and then incubated with poly(MVE-MA)-coated magnetic beads. After magnetic separation of the beads, Quick Navi-Adeno was used for the detection of adenovirus hexon protein by immunochromatography. The presence of adenovirus hexon protein was interpreted on the basis of the presence or absence of a test line (T). A positive control was also included (C). Samples were divided into the following categories: BD, sample before incubation with the beads (BF), supernatant after incubation with the beads (SP), and samples containing the same quantity of adenovirus as in the BD (total fraction, TL). All samples were solubilized with lysis buffer and subjected to immunochromatography.

Abbreviations: BD, bead fraction; PBS, phosphate-buffered solution; poly(MVE-MA), poly(methyl vinyl ether-maleic anhydrate).

the BD fraction, a band of lower intensity was detected in the sample before incubation with the beads (BF fraction). To further examine whether this magnetic capture method can be applied to different serotypes of adenovirus, Adenoid75 (Adenovirus Type 5), Tonsil99 (Adenovirus Type 6), Slobitski (Adenovirus Type 11), AV-587[3911] (Adenovirus Type 19), and Tak(73-3544) (Adenovirus Type 41) were subjected to the capture procedure. Our results showed that all types of adenoviruses could be efficiently captured by the anionic magnetic beads (Figure 3). The band intensities obtained by immunochromatography (Figure 3) were then compared using ImageJ software. The band intensity corresponding to TL for each type of adenovirus was taken as 1 . Band intensities for BD, BF, and SP are listed in Table 1.

Table I The band intensities of immunochromatography in each fraction from various strains of adenovirus captured by the anionic polymer-coated magnetic beads

\begin{tabular}{lllll}
\hline Strains & BD & SP & BF & $\begin{array}{l}\text { Concentrating rate } \\
\text { (BD/BF) }\end{array}$ \\
\hline Adenoid75 & 0.842 & 0 & 0.228 & 3.7 \\
Tonsil99 & 0.859 & 0 & 0.406 & 2.1 \\
Slobitski & 1.074 & 0 & 0.037 & 29.0 \\
AV-587[39II] & 0.932 & 0 & 0.295 & 3.2 \\
Tak(73-3544) & 0.900 & 0.02 & 0.280 & 3.2 \\
\hline
\end{tabular}

Notes: The band intensities of each fraction shown in immunochromatography using various strains of adenovirus were compared using Imagej software. The band intensity of TL for each strain of adenovirus was taken as I. Band intensities of each fraction (BD, SP, and BF) compared to TL are listed. Concentrating rate was calculated by the intensity in BD divided by the intensity in BF. Samples were divided into the following: beads fraction (BD), sample before incubation with the beads $(\mathrm{BF})$, supernatant after incubation with the beads (SP), and sample containing the same quantity of adenovirus as in the BD (total fraction, $T L$ ).
The data indicate that the beads had concentrated the adenovirus 3.7-fold for Adenoid75, 2.1-fold for Tonsil99, 29.0fold for Slobitski, 3.2-fold for AV-587[3911], and 3.2-fold for $\operatorname{Tak}(73-3544)$. The capture efficiencies were $84.2 \%$ for Adenoid75, 85.9\% for Tonsi199, 100\% for Slobitski, 93.2\% for AV-587[3911], and 90.0\% for Tak(73-3544).

We also carried out similar capture experiments using clinical nasal aspirates (Figure 4). Here, a band corresponding to adenovirus hexon protein was detected in BD and TL but not in SP and BF samples, demonstrating the successful capture of adenovirus from nasal aspirates containing a low quantity of virus particles.

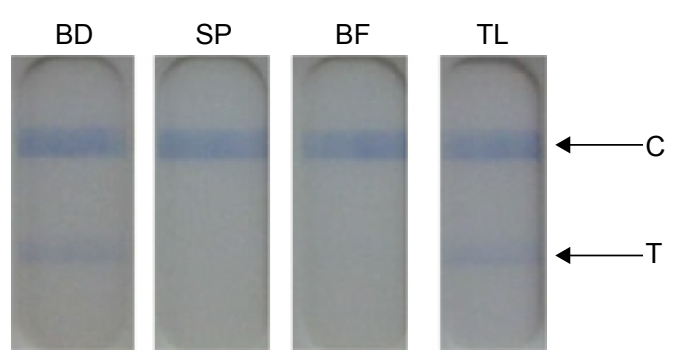

Figure 4 Detection of hexon protein of adenovirus adsorbed onto anionic polymercoated magnetic beads from a NAS.

Notes: Adenovirus in adenovirus-infected NAS was diluted with PBS and subjected to incubation with poly(MVE-MA)-coated magnetic beads. Quick Navi-Adeno was used for the detection of adenovirus hexon protein by immunochromatography. The presence of adenovirus hexon protein was interpreted on the basis of the presence and absence of a test line (T). A positive control was also included (C). Samples were divided into the following categories: $\mathrm{BD}$, sample before incubation with the beads (BF), supernatant after incubation with the beads (SP), and samples containing the same quantity of adenovirus as in the $\mathrm{BD}$ (total fraction, $\mathrm{TL}$ ). All samples were solubilized with lysis buffer and subjected to immunochromatography.

Abbreviations: BD, bead fraction; NAS, nasal aspirate; PBS, phosphate-buffered solution; poly(MVE-MA), poly(methyl vinyl ether-maleic anhydrate). 


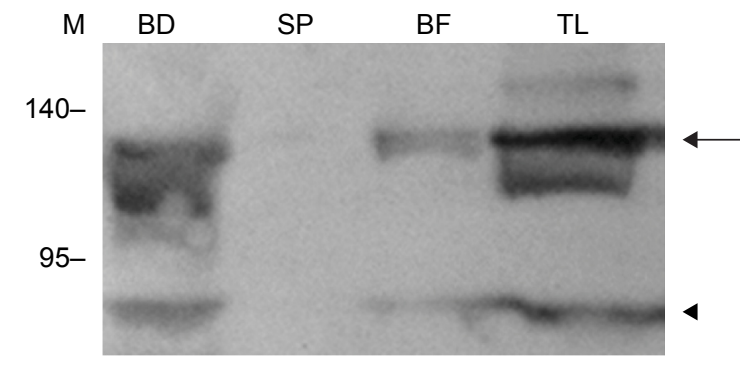

Figure 5 Detection of viral proteins in adenovirus recovered with anionic polymercoated magnetic beads using a Western blotting procedure.

Notes: Adenovirus AxCAwt2 $(20 \mu \mathrm{L})$ diluted with PBS was captured using poly(MVE-MA)-coated magnetic beads. Samples were divided into three categories: $B D$, supernatant after incubation with the beads (SP), and sample before incubation with the beads (BF). Samples containing the same quantity of adenovirus as in the BD (total fraction, TL) was also prepared. All samples were solubilized in SDS-gel loading buffer and resolved by SDS-PAGE using an $8 \%$ gel. Samples were then analyzed by Western blotting using a polyclonal antiadenovirus antibody. Bands corresponding to hexon (arrow) and penton (arrowhead) protein are highlighted. The molecular mass markers $(M)$ are shown on the left-hand side of the blot. Numbers on the left are molecular weight $(\mathrm{kDa})$

Abbreviations: BD, bead fraction; PBS, phosphate-buffered solution; poly(MVE-MA), poly(methyl vinyl ether-maleic anhydrate); PAGE, polyacrylamide gel electrophoresis; SDS, sodium dodecyl sulfate.

Next, we investigated whether the bead-captured adenovirus could be detected using a Western blotting procedure (Figure 5). Two bands with a molecular mass of $\sim 120$ and $80 \mathrm{kDa}$ were detected, corresponding to hexon protein $(116 \mathrm{kDa})$ and penton protein $(80 \mathrm{kDa})$, respectively. ${ }^{25}$ ImageJ software was used to compare band intensities in the hexon region between the $\mathrm{BD}$ and $\mathrm{TL}$ fractions from eight independent experiments. These results showed the value for BD represents $73 \% \pm 5.7 \%$ compared to TL (100\%).

Next, we investigated whether the captured adenovirus retained infectivity after bead separation. Fractions from the cell medium of HEK293 cells transfected with recombinant adenovirus vector AxCAwt 2 were subjected to viral titration assays using HEK293 cells (Figure 6). The results showed a similar viral titer $(\mathrm{PFU} / \mathrm{mL})$ for the $\mathrm{BD}$ and $\mathrm{TL}$ fractions. By contrast, the BF fraction had a viral titer of one-tenth that of the BD and TL fractions. Moreover, the viral titer of the SP fraction was below the detection limit for our assay procedure. These observations were further supported by X-gal staining of HEK293 cells transfected with AxCAiLacZ (Figure 7) and enzyme-linked immunosorbent assay (ELISA) using an antiadenovirus hexon antibody (Figure 8). Specifically, X-gal was found to stain cells treated with samples of BD and TL, but not SP (Figure 7). After incubation of HEK293 cells with the TL and BD fractions, adenovirus infected cells were identified by ELISA. However, no adenovirus infected cells were found after incubation with the SP fraction (Figure 8). These results suggest that the vast majority of infectious adenovirus was efficiently captured from solution by the anionic magnetic beads.

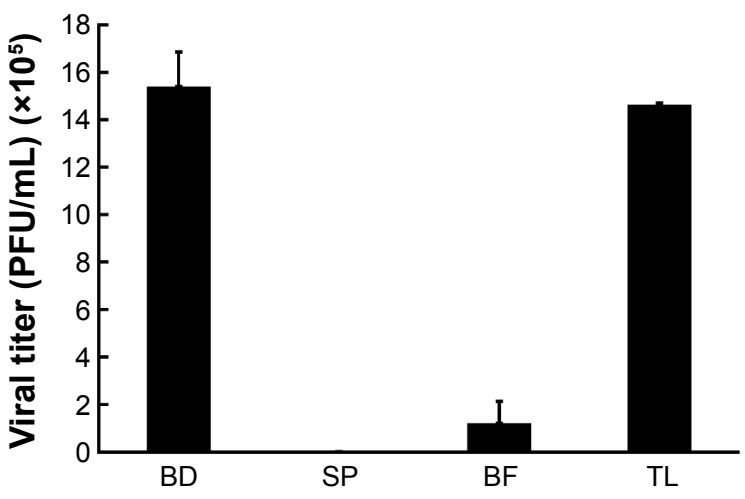

Figure 6 Virus titration assay showed recovery of infectious viral particles from the anionic polymer-coated magnetic beads.

Notes: Cell culture medium of HEK293 cells transfected with adenovirus vector AxCAwt2 was diluted with PBS and the virus particles were then captured using poly(MVE-MA)-coated magnetic beads. The resultant BD, supernatant after incubation with the beads (SP), sample before incubation with the beads (BF), and samples containing the same quantity of adenovirus as in the BD (total fraction, $T L$ ) were subjected to a viral titer assay (PFU/mL).

Abbreviations: BD, bead fraction; PBS, phosphate-buffered solution; poly(MVE-MA), poly(methyl vinyl ether-maleic anhydrate); PFU, plaque forming units.

Next, we examined the capacity of the anionic magnetic beads to capture adenovirus using PCR to detect the adenovirus genomic DNA (Figure 9). The amplified PCR product was anticipated to be $1,597 \mathrm{bp}$ in length corresponding to the adenovirus hexon gene. A single band of the expected size was amplified from the BD and from the same quantity of cell culture medium as in the BD fraction (TL) using cell culture medium from AxCAwt2 vector-transfected HEK293 cells. No amplified product was detected in the supernatant after incubation with the beads (SP). The amplified product obtained from both the $\mathrm{BD}$ and TL fractions was confirmed to be the hexon gene of adenovirus Type 5 by DNA sequence analysis (ie, identity to Genbank accession number AF542116.1 was 95\% using the forward primer and $97 \%$ using the reverse primer).

We also measured the amount of adenovirus genomic DNA in the BD, SP, and TL fractions by real-time PCR of the hexon gene, relative to that of a control (BF sample with the highest value was taken as 1; Figure 10). By comparison to TL $(100 \%)$, the amount of viral DNA detected by real-time PCR in the cell culture medium of HEK293 cells transfected with AxCAiLacZ was determined to be $83.33 \%$ for the BD fraction and $5.39 \%$ for the $\mathrm{BF}$ fraction. The specificity of the PCR was confirmed by dissociation curve analysis of the reaction products. The $140 \mathrm{bp}$ band obtained by real-time PCR was also confirmed to correspond to the hexon gene of adenovirus Type 5 by DNA sequencing (identity to Genbank accession number AF542116.1 was 94\% using the forward primer and 96\% using the reverse primer). Taken together, 


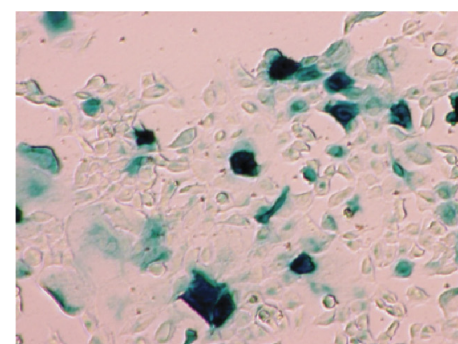

TL

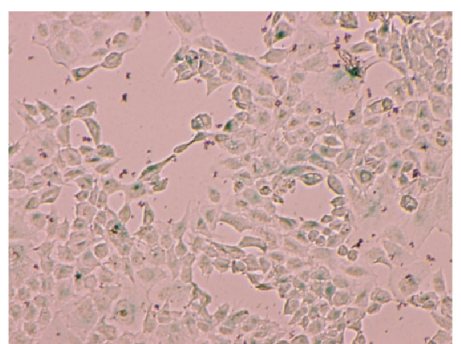

SP

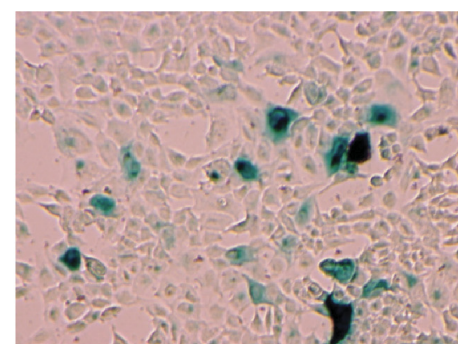

BD

Figure 7 X-gal staining showed recovery of infectious viral particles using anionic polymer-coated magnetic beads.

Notes: Cell culture medium of HEK293 cells transfected with adenovirus vector AxCAiLacZ was diluted with PBS and the virus particles were then captured using poly(MVE-MA)-coated magnetic beads. The resultant BD, supernatant after incubation with the beads (SP), and samples containing the same quantity of adenovirus as in the $B D$ (total fraction, $T L$ ) were subjected to $X$-gal staining.

Abbreviations: BD, bead fraction; PBS, phosphate-buffered solution; poly(MVE-MA), poly(methyl vinyl ether-maleic anhydrate).

these results showed that all of the adenovirus in the cell culture medium could be captured by the magnetic beads. Indeed, all of the analyzed components of adenovirus were identified within the magnetic bead-captured fraction.

Finally, we examined whether our capture method enhances the sensitivity of adenovirus detection by immunochromatography. Thus, a solution containing adenovirus below the detection limit for immunochromatography was subjected to the capture procedure. Serial $(\times 2)$ dilutions were prepared of an adenovirus-containing solution with a titer varying from $4.0 \times 10^{3} \mathrm{PFU} / \mu \mathrm{L}$ to $2.5 \times 10^{2} \mathrm{PFU} / \mu \mathrm{L}$. Immunochromatography (Quick Navi-Adeno) successfully detected adenovirus down to a level of $1.0 \times 10^{3} \mathrm{PFU} / \mu \mathrm{L}$ but not $5.0 \times 10^{2} \mathrm{PFU} / \mu \mathrm{L}$ (data not shown). Adenovirus with a titer of $5.0 \times 10^{2} \mathrm{PFU} / \mu \mathrm{L}$ was subjected to the virus capture procedure using the magnetic beads. Immunochromatography was then used to detect adenovirus before and after the bead concentrating procedure (Figure S1). Our results showed that a viral titer of $5.0 \times 10^{2} \mathrm{PFU} / \mu \mathrm{L}$ could be detected by immunochromatography (Quick Navi-Adeno) only after concentration with the beads.

\section{Discussion}

The magnetic bead-mediated capture method for adenovirus described in this paper is a simple and quick procedure that significantly enhances the detection of adenoviruses. The overall processing time of this method is approximately 30 minutes, which is considerably quicker than existing methodologies. Moreover, complicated procedures such as column purification are not required. The results from this study suggest that the magnetic bead capture method can identify adenovirus in samples below the current detection limit. In addition, the relatively small number of processing steps reduces problems associated with loss of signal and potential cross-contamination commonly observed with multistep protocols. The magnetic bead-mediated capture method is also fully compatible with conventional detection methods of immunochromatography such as PCR, ELISA, and Western blotting. Indeed, alternative methods for concentrating viruses are often found to be incompatible with conventional detection procedures. ${ }^{13-15}$ For example, PEG precipitation is sometimes incompatible with PCR due to

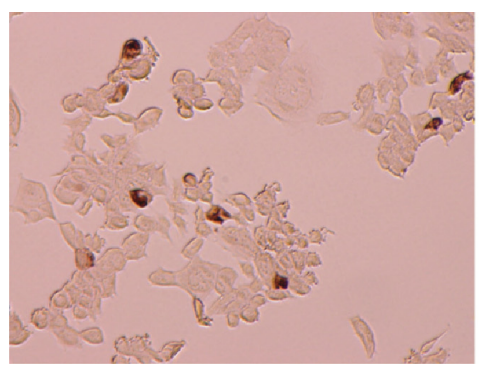

TL

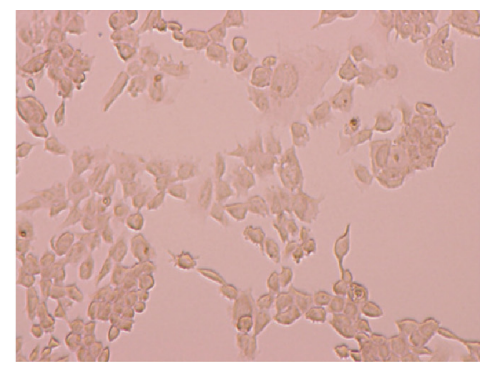

SP

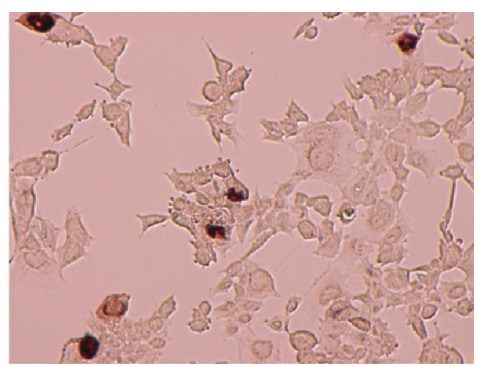

BD

Figure 8 ELISA showed recovery of infectious viral particles using anionic polymer-coated magnetic beads.

Notes: Cell culture medium of HEK293 cells transfected with adenovirus vector AxCAwt2 was diluted with PBS and the virus particles were then captured using poly(MVEMA)-coated magnetic beads. The resultant BD, supernatant after incubation with the beads (SP), and samples containing the same quantity of adenovirus as in the BD (total fraction, TL) were subjected to ELISA using antiadenovirus hexon protein antibody.

Abbreviations: BD, bead fraction; ELISA, enzyme-linked immunosorbent assay; PBS, phosphate-buffered solution; poly(MVE-MA), poly(methyl vinyl ether-maleic anhydrate). 


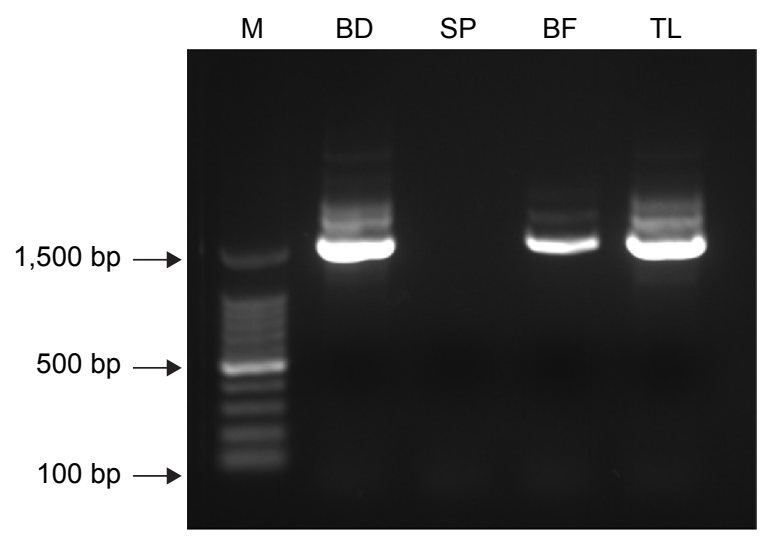

Figure 9 Detection of the DNA genome of adenovirus adsorbed onto anionic polymer-coated magnetic beads.

Notes: Adenovirus AxCAwt2 $(20 \mu \mathrm{L})$ in $500 \mu \mathrm{L}$ PBS were mixed with poly(MVEMA)-coated magnetic beads. After incubation, the following fractions were obtained: $B D$, supernatant after incubation with the beads (SP), sample before incubation with the beads (BF), and samples containing the same quantity of adenovirus as in the $B D$ (total fraction, TL). Viral genomic DNA was subsequently extracted from the various fractions using a QIAamp Viral RNA mini kit. These experiments confirmed that adenoviral DNA could be extracted from the beads and subjected to the PCR. DNA corresponding to the adenovirus hexon gene (1597-bp) was amplified by PCR. The identity of the amplified products was confirmed by DNA sequencing. The lefthand lane is size marker (M), which includes DNA of $100,200,300,400,500,600$, $700,800,900,1,000,1,200$, and I,500 bp. The positions of the $100 \mathrm{bp}, 500 \mathrm{bp}$, and I,500 bp bands are indicated by arrows.

Abbreviations: BD, bead fraction; PBS, phosphate-buffered solution; poly(MVE$M A)$, poly(methyl vinyl ether-maleic anhydrate); PCR, polymerase chain reaction.

PEG-mediated inhibition of the DNA polymerase used in the amplification step. In addition, the ultracentrifugation procedure requires expensive specialist equipment and is relatively time consuming compared to the magnetic beadmediated capture method. Therefore, compared to previous virus concentrating methods, our capture procedure using

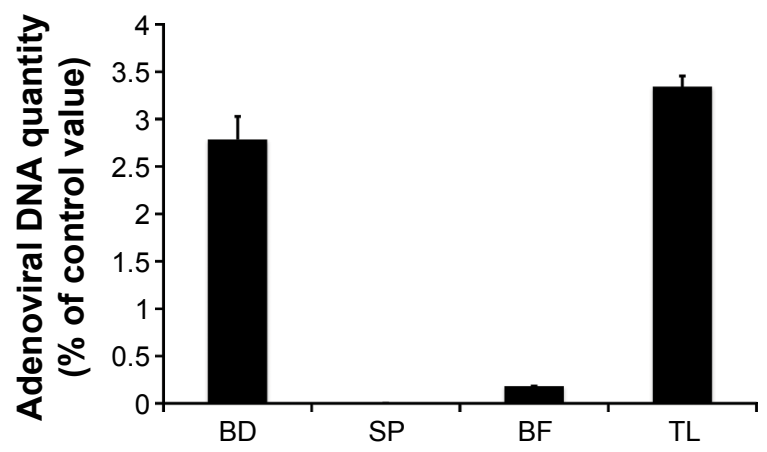

Figure 10 Quantitative analysis of adenovirus DNA adsorbed onto anionic polymercoated magnetic beads.

Notes: The poly(MVE-MA)-coated magnetic beads were used to capture adenovirus from $20 \mu \mathrm{L}$ of culture medium of HEK293 cells transfected with AxCAiLacZ diluted with PBS and then analyzed by real-time PCR using realAdenoHexon-F and realAdenoHexon- $R$ primers for the hexon gene of adenovirus. Samples were divided into four fractions: I) BD, 2) supernatant after incubation with the beads (SP), 3) sample before incubation with the beads (BF), and 4) samples containing the same quantity of adenovirus as in the BD (total fraction, $T L$ ). The BF sample with the highest value was taken as I.

Abbreviations: BD, bead fraction; PBS, phosphate-buffered solution; poly(MVE$M A)$, poly(methyl vinyl ether-maleic anhydrate); $P C R$, polymerase chain reaction. magnetic beads is a promising approach that is well suited to established detection techniques.

There are several methods for concentrating a virus using magnetic beads coated with an antibody for a specific virus. ${ }^{14,26-30}$ Polymers such as polyethyleneimine have been used to concentrate simian virus $40,{ }^{31}$ herpes simplex virus Type $1,{ }^{31}$ Sindbis virus, ${ }^{31}$ vesicular stomatitis virus, ${ }^{31}$ amphotropic murine leukemia virus, ${ }^{32}$ poliovirus, ${ }^{33}$ hepatitis A virus,${ }^{33}$ hepatitis B virus, ${ }^{33}$ hepatitis C virus, ${ }^{33}$ and cytomegalovirus (CMV). ${ }^{34}$ Sulfonated magnetic beads in the presence of divalent cations are known to concentrate CMV, ${ }^{34}$ Sindbis virus ${ }^{34}$ poliovirus, ${ }^{34}$ and porcine parvovirus. ${ }^{34}$ Moreover, poly(MVE-MA)-coated magnetic beads can be used for the efficient capture of avian and human influenza virus, ${ }^{17,21}$ RSV, ${ }^{19}$ Borna disease virus, ${ }^{20}$ dengue virus, ${ }^{22} \mathrm{HIV}-1,{ }^{16,18}$ CMV,${ }^{16}$ herpesvirus, ${ }^{35}$ and vaccinia virus. ${ }^{35}$

This study clearly shows that poly(MVE-MA)-coated magnetic beads can be used to capture adenovirus in various sample types, including cell culture medium and nasal aspirates. Nonetheless, further work is required to demonstrate the ability of the magnetic beads to specifically concentrate adenovirus from a mixture of viruses present in a biological sample. Indeed, a previous study using RSV showed that the binding of RSV to the magnetic beads is abrogated in a competitive manner by the presence of influenza $\mathrm{A}$ and $\mathrm{B}$ virus. ${ }^{19}$ This observation suggests that the binding mechanism is the same for these viruses. Thus, further studies are required to clarify whether this is also the case for adenovirus.

Previous studies using RSV and HIV-1 have shown that preincubation of the beads with neutralizing antibody against Env of the respective virus prevents capture. ${ }^{18,19}$ These findings suggested Env may be involved in the binding process between the magnetic beads and viral particles. However, this study demonstrates that the nonenveloped adenovirus also binds to the magnetic beads, showing the envelope is not necessarily required for binding.

Given that poly(MVE-MA) is a negatively charged molecule, modification of the spatial organization of this polymer may alter its binding efficiency/capacity for viral particles. Previous studies reported that the surface of adenovirus is also highly negatively charged. ${ }^{36}$ Thus, an anionic charge on the polymer might not be preferable for adenovirus binding. However, other functions of the polymer besides its charge may contribute to the binding of adenovirus. This idea is supported by the observation that adenovirus binding to the beads was not influenced by different $\mathrm{pH}$ conditions (Figure S2). Therefore, it is assumed that the mechanism for adenovirus binding is not dependent on charge. However, our previous studies using RSV have shown that a slightly 
lower level of virus binding to the magnetic beads is observed under high $\mathrm{pH}$ conditions. ${ }^{19}$ Thus, the contribution of charge to the binding mechanism may vary between viruses. Further analysis of the effect of charge density and steric spatial organization of poly(MVE-MA) on the binding to various types of virus may help elucidate the mechanism of interaction. In addition, information on the exact size and charge values of the polymer on the beads would also be important for understanding binding mechanisms. To test this hypothesis, further studies are required to examine whether changes to the spatial organization and length of poly(MVE-MA) alter the binding to the viruses.

Of the different serotypes of adenovirus analyzed in this study, Type1 Slobitski was most effectively bound by the beads (Table 1). This observation implies that different adenovirus types may have different binding capacities to the beads. A previous study has shown varying $\mathrm{pH}$ stabilities among different types of adenovirus. ${ }^{37}$ Moreover, the stability of adenovirus penton protein is known to be strongly $\mathrm{pH}$ dependent. ${ }^{3}$ Thus, binding analysis using a combination of various adenovirus types under different $\mathrm{pH}$ conditions may also help to elucidate the mechanisms by which adenoviruses bind to the beads.

A number of factors that inhibit binding between the magnetic beads and viruses have been reported. ${ }^{17}$ These inhibitory factors include blood and albumin, which nonspecifically bind to the beads and reduce their binding capacity. Clinical samples are often contaminated with inhibitory factors, especially from blood. Thus, a strategy for reducing the inhibitory effect of these factors would be advantageous. Modification of the charge density and surface organization of the anionic polymers on the magnetic beads may be effective in reducing nonspecific binding.

We have demonstrated a simple and straightforward procedure involving magnetic beads coated with anionic polymers that can efficiently capture and concentrate adenovirus. Viral genomic DNA as well as the major capsid proteins hexon and penton were detected in the captured samples either using PCR, real-time PCR, immunochromatography, or Western blotting. Therefore, our capture method is fully compatible with conventional means of viral detection. Finally, our novel method enables early and sensitive detection of adenovirus, which can potentially improve the therapeutic outcome where currently only palliative treatment is possible.

\section{Acknowledgments}

The authors thank Mr Satoru Shigeno and Mr Shinya Iriguchi for technical assistance. This work was supported by a Grant-in-Aid for the Promotion of Basic Research Activities for Innovative Biosciences from Bio-oriented Technology Research Advancement Institution (BRAIN) and the Japan Science and Technology Agency (JST) as well as a Grant-in-Aid for Scientific Research on Innovative Areas from the Japan Society for the Promotion of Science and a Grant-in-Aid from Science and Technology Research Promotion Program for Agriculture, Forestry, Fisheries, and Food Industry.

\section{Disclosure}

The authors report no conflicts of interest in this work.

\section{References}

1. Horwitz MS. Adenoviruses. In: Knipe DM, Howley PM, Griffin DE, Lamb RA, Martin MA, Roizman B, Straus SE, editors. Fields Virology. 4th ed. Philadelphia, PA: Lippincott Williams \& Wilkins; 2001: 2301-2326.

2. Fong TT, Phanikumar MS, Xagoraraki I, Rose JB. Quantitative detection of human adenoviruses in wastewater and combined sewer overflows influencing a Michigan river. Appl Environ Microbiol. 2010;76(3):715-723.

3. Russell WC. Adenoviruses: update on structure and function. J Gen Virol. 2009;90(Pt 1):1-20.

4. Ishiko H, Shimada Y, Konno T, et al. Novel human adenovirus causing nosocomial epidemic keratoconjunctivitis. J Clin Microbiol. 2008; 46(6):2002-2008.

5. Echavarria M. Adenoviruses in immunocompromised hosts. Clin Microbiol Rev. 2008;21(4):704-715.

6. Echavarria M, Maldonado D, Elbert G, Videla C, Rappaport R, Carballal G. Use of PCR to demonstrate presence of adenovirus species $\mathrm{B}, \mathrm{C}$, or $\mathrm{F}$ as well as coinfection with two adenovirus species in children with flu-like symptoms. J Clin Microbiol. 2006;44(2):625-627.

7. Morozumi M, Shimizu H, Matsushima Y, et al. Evaluation of new immunochromatographic assay kit for adenovirus detection in throat swab: comparison with culture and real-time PCR results. $J$ Infect Chemother. 2014;20(5):303-306.

8. Kim J, Kim HS, Kim JS, et al. Evaluation of an immunochromatographic assay for the rapid and simultaneous detection of rotavirus and adenovirus in stool samples. Ann Lab Med. 2014;34(3):216-222.

9. Romero-Gómez MP, López López R, González Montes R, et al. Immunochromatographic test for detection of adenovirus from respiratory samples: is it a real solution for pediatric emergency department? J Virol Methods. 2014;195:236-239.

10. Kittigul L, Khamoun P, Sujirarat D, et al. An improved method for concentrating rotavirus from water samples. Mem Inst Oswaldo Cruz. 2001;96(6):815-821.

11. Sanyal D, Kudesia G, Corbitt G. Comparison of ultracentrifugation and polyethylene glycol precipitation for concentration of hepatitis B virus (HBV) DNA for molecular hybridisation tests and the relationship of HBV-DNA to $\mathrm{HBe}$ antigen and anti-HBe status. $J$ Med Microbiol. 1991;35(5):291-293.

12. Trépanier $P$, Payment $P$, Trudel M. Concentration of human respiratory syncytial virus using ammonium sulfate, polyethylene glycol or hollow fiber ultrafiltration. J Virol Methods. 1981;3(4):201-211.

13. Roth WK, Weber M, Seifried E. Feasibility and efficacy of routine PCR screening of blood donations for hepatitis $\mathrm{C}$ virus, hepatitis $\mathrm{B}$ virus, and HIV-1 in a blood-bank setting. Lancet. 1999;353(9150):359-363.

14. Kobayashi S, Natori K, Takeda N, Sakae K. Immunomagnetic capture rt-PCR for detection of norovirus from foods implicated in a foodborne outbreak. Microbiol Immunol. 2004;48(3):201-204.

15. Novotný J, Svobodová J, Ransnäs LA, Kubistová K. A method for the preparation of purified antigens of coxsackievirus B3 from a large volume of cell culture supernatant. Acta Virol. 1992;36(5):483-487. 
16. Flavigny E, Gaboyard M, Merel P, Fleury H. Magnetic particlemediated virus concentration for clinical virology. Poster presented at: 104th General Meeting of the American Society for Microbiology, New Orleans, American Society for Microbiology, May 22-27, 2004, Washington, DC.

17. Sakudo A, Baba K, Tsukamoto M, et al. Anionic polymer, poly(methyl vinyl ether-maleic anhydride)-coated beads-based capture of human influenza A and B virus. Bioorg Med Chem. 2009;17(2):752-757.

18. Sakudo A, Ikuta K. A technique for capturing broad subtypes and circulating recombinant forms of HIV-1 based on anionic polymer-coated magnetic beads. Int J Mol Med. 2012;30(2):437-442.

19. Sakudo A, Baba K, Tsukamoto M, Ikuta K. Use of anionic polymer, poly(methyl vinyl ether-maleic anhydride)-coated beads for capture of respiratory syncytial virus. Bioorg Med Chem Lett. 2009;19(15): 4488-4491.

20. Sakudo A, Tanaka Y, Ikuta K. Capture of infectious borna disease virus using anionic polymer-coated magnetic beads. Neurosci Lett. 2011;494(3): 237-239.

21. Sakudo A, Ikuta K. Efficient capture of infectious H5 avian influenza virus utilizing magnetic beads coated with anionic polymer. Biochem Biophys Res Commun. 2008;377(1):85-88.

22. Sakudo A, Masrinoul P, Tanaka Y, Ikuta K. Capture of dengue virus type 3 using anionic polymer-coated magnetic beads. Int J Mol Med.2011; 28(4):625-628.

23. Baba K, Inventor; Kochi-kai. Virus collecting instrument. Japan Patent 2008-119552. May 29, 2008.

24. Mitamura K, Shimizu H, Yamazaki M, et al. Evaluation of new rapid diagnosis kit for adenovirus, Quicknavi ${ }^{\mathrm{TM}}$-Adeno. Jpn J Med Pharm Sci. 2008;60:143-150.

25. Tuteja U, Batra HV. Generation and characterization of monoclonal antibodies to adenovirus. Indian J Exp Biol. 2000;38(12):1259-1262.

26. Clavet CR, Margolin AB, Regan PM. Herpes simplex virus type- 2 specific glycoprotein G-2 immunomagnetically captured from HEp-2 infected tissue culture extracts. J Virol Methods. 2004;119(2):121-128.

27. Jothikumar N, Cliver DO, Mariam TW. Immunomagnetic capture PCR for rapid concentration and detection of hepatitis A virus from environmental samples. Appl Environ Microbiol. 1998;64(2):504-508.
28. Sakudo A, Chou H, Nagatsu M. Antibody-integrated and functionalized graphite-encapsulated magnetic beads, produced using ammonia gas plasma technology, for capturing Salmonella. Bioorg Med Chem Lett. 2015;25(5):1012-1016.

29. Sakudo A, Chou H, Ikuta K, Nagatsu M. Integration of antibody by surface functionalization of graphite-encapsulated magnetic beads using ammonia gas plasma technology for capturing influenza A virus. Bioorg Med Chem Lett. 2015;25(9):1876-1879.

30. Sakudo A, Viswan A, Chou H, Sasaki T, Ikuta K, Nagatsu M. Capture of dengue viruses using antibody-integrated graphite encapsulated magnetic beads produced by gas plasma technology. Mol Med Rep, in press.

31. Satoh K, Iwata A, Murata M, Hikata M, Hayakawa T, Yamaguchi T. Virus concentration using polyethyleneimine-conjugated magnetic beads for improving the sensitivity of nucleic acid amplification tests. J Virol Methods. 2003;114(1):11-19.

32. Uchida E, Sato K, Iwata A, et al. An improved method for detection of replication-competent retrovirus in retrovirus vector products. Biologicals. 2004;32(3):139-146.

33. Uchida E, Kogi M, Oshizawa T, et al. Optimization of the virus concentration method using polyethyleneimine-conjugated magnetic beads and its application to the detection of human hepatitis A, B and $\mathrm{C}$ viruses. $J$ Virol Methods. 2007;143(1):95-103.

34. Iwata A, Satoh K, Murata M, Hikata M, Hayakawa T, Yamaguchi T. Virus concentration using sulfonated magnetic beads to improve sensitivity in nucleic acid amplification tests. Biol Pharm Bull. 2003;26(8):1065-1069.

35. Hatano B, Kojima A, Sata T, Katano H. Virus detection using ViroAdembeads, a rapid capture system for viruses, and plaque assay in intentionally virus-contaminated beverages. Jpn J Infect Dis. 2010;63(1): $52-54$.

36. Prazeres DMF, Santos JAL. Production and purification of adenovirus vectors for gene therapy. In: Gad SC, editor. Handbook of Pharmaceutical Biotechnology. Hoboken, NJ: Wiley-Interscience; 2006: 1261-1295.

37. Rafajko RR, Young JC. Thermal and $\mathrm{pH}$ stability of adenovirus types 12, 14, AND 18. Proc Soc Exp Biol Med. 1964;116:683-685. 


\section{Supplementary materials}

BF

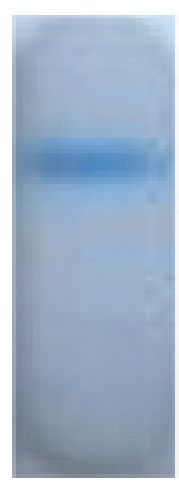

BD

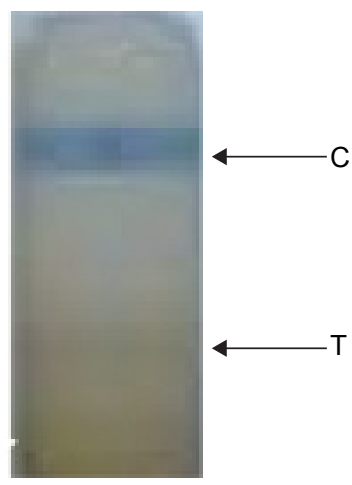

Figure SI The concentrating procedure using anionic polymer-coated magnetic beads increases the sensitivity of immunochromatography for the detection of adenovirus. Notes: Samples containing adenovirus below the detection limit for immunochromatography (Quick Navi-Adeno) $\left(5.0 \times 10^{2} \mathrm{PFU} / \mu \mathrm{L}\right)$ were subjected to magnetic separation using poly(MVE-MA)-coated magnetic beads. The BD was then solubilized with lysis buffer and subjected to a conventional immunochromatography procedure. The presence of adenovirus hexon protein was interpreted on the basis of the presence or absence of a test line (T). A positive control was also included (C). Our results show the incorporation of the magnetic bead concentrating step enables the identification of adenovirus by immunochromatography in samples that would otherwise be below the detection limit for this method.

Abbreviations: BD, bead fraction; poly(MVE-MA), poly(methyl vinyl ether-maleic anhydrate); BF, supernatant after incubation with the beads; PFU, plaque forming units.

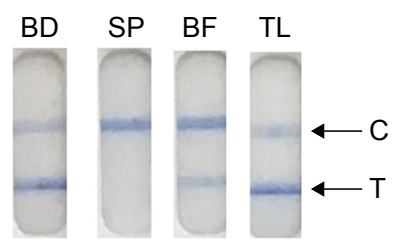

pH 6.0
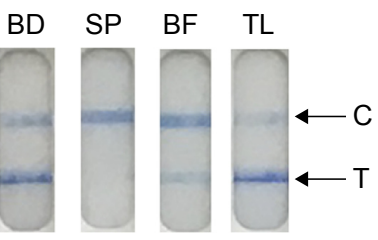

pH 6.5
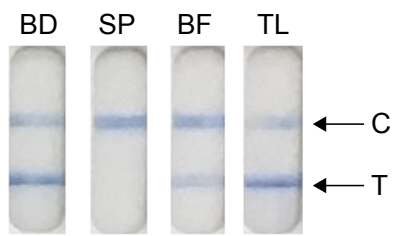

pH 7.0

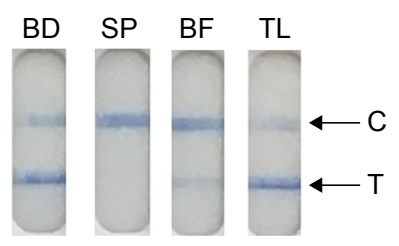

$\mathrm{pH} 7.5$
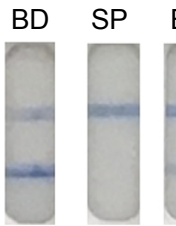

$\mathrm{pH} 8.0$

Figure S2 Adsorption of adenovirus onto anionic polymer-coated magnetic beads under different pH conditions.

Notes: Recombinant adenovirus Axcw2 (20 $\mu \mathrm{L}$ ) was diluted with $500 \mu \mathrm{L}$ of HEPES (4-(2-hydroxyethyl)-I-piperazineethanesulfonic acid) buffer (pH 6.0-8.0), which was prepared by adding different volumes of $0.1 \mathrm{M} \mathrm{NaOH}$ to $0.1 \mathrm{M}$ HEPES solution, and then incubated with poly(MVE-MA)-coated magnetic beads. After magnetic separation of the beads, Quick Navi-Adeno was used for the detection of adenovirus hexon protein by immunochromatography. The presence of adenovirus hexon protein was interpreted on the basis of the presence or absence of a test line ( $T$ ). A positive control was also included (C). Samples were divided into the following categories: BD, sample before incubation with the beads (BF), supernatant after incubation with the beads (SP), and samples containing the same quantity of adenovirus as in the BD (total fraction, $T L)$. All samples were solubilized with lysis buffer and subjected to immunochromatography.

Abbreviations: BD, bead fraction; poly(MVE-MA), poly(methyl vinyl ether-maleic anhydrate); HEPES, (4-(2-hydroxyethyl)-I-piperazineethanesulfonic acid).

International Journal of Nanomedicine

\section{Publish your work in this journal}

The International Journal of Nanomedicine is an international, peerreviewed journal focusing on the application of nanotechnology in diagnostics, therapeutics, and drug delivery systems throughout the biomedical field. This journal is indexed on PubMed Central, MedLine, CAS, SciSearch $\AA$, Current Contents ${ }^{\circledR} /$ Clinical Medicine,

\section{Dovepress}

Journal Citation Reports/Science Edition, EMBase, Scopus and the Elsevier Bibliographic databases. The manuscript management system is completely online and includes a very quick and fair peer-review system, which is all easy to use. Visit http://www.dovepress.com/ testimonials.php to read real quotes from published authors. 